\title{
On Symmetric Solutions of the Relativistic Vlasov-Poisson System*
}

\author{
Robert T. Glassey ${ }^{1}$ and Jack Schaeffer ${ }^{2}$
}

1 Department of Mathematics, Indiana University, Bloomington, IN 47405, USA

2 Department of Mathematics, Carnegie-Mellon University, Pittsburgh, PA 15213, USA

\begin{abstract}
Spherically symmetric solutions to the Cauchy problem for the relativistic Vlasov-Poisson system are studied in three space dimensions. If the energy is positive definite (the plasma physics case), global classical solutions exist. In the case of indefinite energy, "small" radial solutions exist in the large, but "large" data solutions (those with negative energy) will blow-up in finite time.
\end{abstract}

\section{Introduction}

The motion of a mono-charged collisionless plasma is described by the VlasovMaxwell (VM) system of equations:

$$
\text { (VM) }\left\{\begin{array}{l}
f_{t}+v \cdot \nabla_{x} f+\gamma(E+v \times B) \cdot \nabla_{v} f=0 \\
E_{t}=\nabla \times B-j \\
B_{t}=-\nabla \times E \\
\nabla \cdot E=\rho, \quad \nabla \cdot B=0 .
\end{array}\right.
$$

Here $E$ and $B$ are the Maxwell electric and magnetic fields and $f=f(x, v, t)\left(x \in \mathbb{R}^{3}\right.$, $v \in \mathbb{R}^{3}, t \geqq 0$ ) is a scalar function describing the density in phase space. The charge and current densities are given by

$$
\begin{aligned}
& \rho=\rho(x, t)=\int_{\mathbb{R}^{3}} f(x, v, t) d v, \\
& j=j(x, t)=\int_{\mathbb{R}^{3}} v f(x, v, t) d v,
\end{aligned}
$$

and $\gamma= \pm 1$. The case $\gamma=+1$ is the plasma physics case, and $\gamma=-1$ is the stellar dynamics case.

The Cauchy problem is to solve (VM) for all $t>0$ with given initial values for $E$,

* Research supported in part by NSF MCS 8319944 
$B$, and $f$. At present, only a local (in time) solution is known [14]. A much simpler system is obtained by assuming that $B$ is identically zero. This is the Vlasov-Poisson system (VP), which takes the form:

$$
\text { (VP) }\left\{\begin{array}{l}
f_{t}+v \cdot \nabla_{x} f+\gamma E \cdot \nabla_{v} f=0 \\
E=\nabla_{x} u, \quad \Delta u=\rho .
\end{array}\right.
$$

Much more is known about the (VP) system. In particular, in three space dimensions, global weak solutions exist $([1,9])$ for $\gamma= \pm 1$, and global classical solutions exist if the Cauchy data are small enough [2]. Classical solutions also exist in the large for symmetric data, as was shown by Batt [3], Horst [8], and Wollman [13]. The existence of classical solutions for "general" data is still unsettled, as is the uniqueness question for weak solutions. For further background we cite the survey [4].

Classical calculations (cf. [11]) suggest that (VM) and (VP) should be correct only for "low" velocities $v$. If "high" velocities can occur, special relativistic corrections should be introduced (cf. $[11,12])$. These result in the relativistic VlasovMaxwell system (RVM):

$$
(\mathrm{RVM})\left\{\begin{array}{l}
f_{t}+\hat{v} \cdot \nabla_{x} f+\gamma(E+\hat{v} \times B) \cdot \nabla_{v} f=0 \\
E_{t}=\nabla \times B-j \\
B_{t}=-\nabla \times E \\
\nabla \cdot E=\rho, \quad \nabla \cdot B=0
\end{array}\right.
$$

where $\hat{v}=v / \sqrt{1+|v|^{2}}, j=\int_{\mathbb{R}^{3}} \hat{v} f d v$, and $\rho$ is as before. If we assume that $B$ is identically zero in (RVM) then we obtain the relativistic Vlasov-Poisson system (RVP):

$$
(\operatorname{RVP})\left\{\begin{array}{l}
f_{t}+\hat{v} \cdot \nabla_{x} f+\gamma E \cdot \nabla_{v} f=0 \\
E=\nabla_{x} u, \quad \Delta u=\rho .
\end{array}\right.
$$

At first sight, (RVP) seems "better" than its classical version, since $|\hat{v}| \leqq 1$. Thus "higher moment difficulties," well-known in the classical case, will not occur. Moreover, for the same reason we have along characteristics $\left|d x_{i} / d s\right|=\left|\hat{v}_{i}\right| \leqq 1$, and therefore one has causality. These favorable circumstances are diminished somewhat by examination of the total energy integral, which for (RVP) takes the form

$$
\iint_{\mathbb{R}^{6}} \sqrt{1+|v|^{2}} f d v d x+\frac{1}{2} \gamma \int_{\mathbb{R}^{3}}|E|^{2} d x=\text { constant. }
$$

In the plasma physics case $(\gamma=+1)$, it follows from this (see Proposition I below) that $\rho \in L^{4 / 3}\left(\mathbb{R}^{3}\right)$ for fixed $t$. This is worse than the result for (VP) itself, where $\rho \in L^{5 / 3}\left(\mathbb{R}^{3}\right)$. However, we show in Sect. II that Batt's and Wollman's methods $[3,13]$ can be adapted and used to show the existence of global spherically symmetric solutions when $\gamma=+1$. However, when $\gamma=-1$, the situation changes dramatically, in contrast to the classical version. Only "small" radial solutions will exist in the large for (RVP) with $\gamma=-1$ (see Sect. II). Indeed, if $\gamma=-1$ and the initial energy is negative, we show in Sect. III that the life-span of such a radial, classical solution is finite. One may hope then that (RVM) is better behaved than (VM), but only when 
$\gamma=+1$. Even when $\gamma=+1$, we are unable to prove global existence for (RVP) for cylindrically-symmetric data, partly because of the weaker result $\rho \in L^{4 / 3}\left(\mathbb{R}^{3}\right)$. (cf. [8]).

We conclude this section by fixing notation and demonstrating a few elementary results.

In the relativistic Vlasov-Poisson system

$$
(\mathrm{RVP})\left\{\begin{array}{l}
f_{t}+\hat{v} \cdot \nabla_{x} f+\gamma E \cdot \nabla_{v} f=0 \\
E=\nabla_{x} u, \quad \Delta u=\rho
\end{array}\right.
$$

one has $\gamma= \pm 1, \hat{v}=v / \sqrt{1+|v|^{2}}$, and $\rho=\int_{\mathbb{R}^{3}} f d v$. An initial value $f(x, v, 0)=\stackrel{\circ}{f}(x, v)$ is prescribed, and we assume from now on that $\stackrel{\circ}{\mathbb{R}^{3}}$ is smooth, non-negative, and of compact support on $\mathbb{R}^{6}$. Since $E$ is defined by the gradient of $u$, we can assume that $u$ vanishes as $|x| \rightarrow \infty$, and hence

$$
u=\frac{-1}{4 \pi} \int_{\mathbb{R}^{3}} \frac{\rho(y, t)}{|x-y|} d y .
$$

Naturally associated with the first-order equation for $f$ are the characteristics: $s \mapsto X(s, t, x, v), s \mapsto V(s, t, x, v)$ defined as the solutions of the following system of ordinary differential equations:

$$
\begin{aligned}
& \frac{d X}{d s}=\hat{V}=V / \sqrt{1+|V|^{2}} \\
& \frac{d V}{d s}=\gamma E(X, s)
\end{aligned}
$$

with the initial values $X(t, t, x, v)=x, V(t, t, x, v)=v$. Since $f$ is constant along characteristics, we conclude that

$$
f(x, v, t)=\stackrel{\circ}{f}(X(0, t, x, v), V(0, t, x, v)),
$$

and hence $\|f(t)\|_{\infty}=\|\stackrel{\circ}{f}\|_{\infty}<\infty$ by assumption. (By $\|f(t)\|_{\infty}$ we mean $\sup \left\{|f(x, v, t)|: x \in \mathbb{R}^{3}, v \in \mathbb{R}^{3}\right\}$.)

Proposition I. Let $f$ be a classical solution of $(\mathrm{RVP})$ on some time interval $(0, T)$ with nonnegative data $f \in C_{0}^{1}\left(\mathbb{R}^{6}\right)$. Then the following properties hold:

(A) If $f$ vanishes for $|x|>k$, then $f(x, v, t)$ vanishes for $|x|>t+k$ (causality).

(B) The total mass is conserved, i.e., $\iint_{\mathbb{R}^{6}} f d v d x=$ constant $\equiv M$.

(C) The total energy is conserved, i.e.,

$$
\int_{\mathbb{R}^{3}}\left\langle\int_{\mathbb{R}^{3}} \sqrt{1+|v|^{2}} f d v+\frac{1}{2} \gamma|E|^{2}\right\rangle d x=\text { constant }=\mathscr{E}_{0} .
$$

(D) If $\gamma=+1$, then there exists a constant $C$ (depending on $\|\stackrel{\circ}{f}\|_{\infty}$ and $\mathscr{E}_{0}$ ) such that $\|\rho(t)\|_{4 / 3} \leqq C$ for $0 \leqq t<T$.

Proof. To prove (A) (causality) we apply Eq. (2) to note that $|X(s, t, x, v)-x|=$ $\left|\int_{t}^{s} \hat{V}(\xi, t, x, v) d \xi\right| \leqq|t-s|$. In particular, $|X(0, t, x, v)-x| \leqq t$. Thus whenever $|x|>$ 
$k+t$, we have $|X(0, t, x, v)| \geqq|x|-|X(0, t, x, v)-x|>k$, and so by hypothesis and (3), $f(x, v, t)=f(X(0, t, x, v), V(0, t, x, v))=0$.

(B) follows by simply integrating (RVP) in $v$ and $x$. To prove (C) define the energy density $e(x, t)$ by

$$
e(x, t)=\int_{\mathbb{R}^{3}} \sqrt{1+|v|^{2}} f d v+\frac{1}{2} \gamma|E|^{2} .
$$

Multiplying (RVP) by $\sqrt{1+|v|^{2}}$ and integrating in $v$, we obtain

$$
\frac{\partial}{\partial t} \int \sqrt{1+|v|^{2}} f d v+\int v \cdot \nabla_{x} f d v-\gamma j \cdot E=0
$$

where $j=\int \hat{v} f d v$.

We have defined $E=\nabla u$, where $\Delta u=\rho$. Multiplying by $u$, we have

$$
\int_{\mathbb{R}^{3}}|E|^{2} d x=-\int_{\mathbb{R}^{3}} \rho u d x
$$

and hence

$$
\begin{aligned}
\frac{d}{d t} \int_{\mathbb{R}^{3}}|E|^{2} d x & =-\int_{\mathbb{R}^{3}} \rho_{t} u d x-\int_{\mathbb{R}^{3}} \rho u_{t} d x=-\int_{\mathbb{R}^{3}} \rho_{t} u d x-\int_{\mathbb{R}^{3}} u_{t} \Delta u d x \\
& =-\int_{\mathbb{R}^{3}} \rho_{t} u d x+\frac{1}{2} \frac{d}{d t} \int_{\mathbb{R}^{3}}|E|^{2} d x .
\end{aligned}
$$

Therefore

$$
\frac{1}{2} \frac{d}{d t} \int_{\mathbb{R}^{3}}|E|^{2} d x=-\int_{\mathbb{R}^{3}} \rho_{t} u d x .
$$

Next, integrating (RVP) in $v$, we get the conservation law

$$
\rho_{t}+\nabla_{x} \cdot j=0
$$

It follows that

$$
\frac{1}{2} \frac{d}{d t} \int_{\mathbb{R}^{3}}|E|^{2} d x=-\int_{\mathbb{R}^{3}} \rho_{t} u d x=\int_{\mathbb{R}^{3}} u \nabla_{x} \cdot j d x=-\int_{\mathbb{R}^{3}} j \cdot \nabla_{x} u d x=-\int_{\mathbb{R}^{3}} j \cdot E d x .
$$

Now using this and $\left(4^{\prime}\right)$ we have

$$
\begin{aligned}
\frac{d}{d t} \int_{\mathbb{R}^{3}}\left(\int_{\mathbb{R}^{3}} \sqrt{1+|v|^{2}} f d v+\frac{1}{2} \gamma|E|^{2}\right) d x & =-\int_{\mathbb{R}^{3}}\left(\int_{\mathbb{R}^{3}} v \cdot \nabla_{x} f d v-\gamma j \cdot E\right) d x-\gamma \int_{\mathbb{R}^{3}} j \cdot E d x \\
& =-\int_{\mathbb{R}^{3}} \int_{\mathbb{R}^{3}} \nabla_{x} \cdot(f v) d v d x=0,
\end{aligned}
$$

which proves (C).

The proof of (D) follows simply the method of Horst [8]. Let $\gamma=+1$, then the energy is positive definite. We write $\rho(x, t)=\int_{|v|<R} f d v+\int_{|v|>R} f d v$. Applying Schwarz's 
inequality we have

$$
\begin{aligned}
\int_{|v|<R} f d v & \leqq\left(\int f^{2} d v\right)^{1 / 2}\left(\frac{4 \pi}{3} R^{3}\right)^{1 / 2} \leqq\left(\|f(t)\|_{\infty} \int f d v\right)^{1 / 2}\left(\frac{4 \pi}{3} R^{3}\right)^{1 / 2} \\
& =\left(\frac{1}{2} \rho(x, t)\right)^{1 / 2}\left(\frac{8 \pi}{3}\|\stackrel{\circ}{f}\|_{\infty} R^{3}\right)^{1 / 2} \leqq \frac{1}{2} \rho(x, t)+\frac{8 \pi}{3}\|\stackrel{\circ}{f}\|_{\infty} R^{3}
\end{aligned}
$$

by Young's inequality. Hence

$$
\frac{1}{2} \rho(x, t) \leqq \frac{8 \pi}{3}\|\stackrel{\circ}{f}\|_{\infty} R^{3}+\int_{|v|>R} f d v \leqq \frac{8 \pi}{3}\|\stackrel{\circ}{f}\|_{\infty} R^{3}+R^{-1} \int \sqrt{1+|v|^{2}} f d v
$$

Taking $R=\left(\int \sqrt{1+|v|^{2}} f d v\right)^{1 / 4}$, we get

$$
\rho(x, t) \leqq\left(\frac{16 \pi}{3}\|\stackrel{\circ}{f}\|_{\infty}+2\right)\left(\int \sqrt{1+|v|^{2}} f d v\right)^{3 / 4},
$$

from which (D) follows.

We conclude this section with some notation regarding spherical symmetry. We assume that $f$ is spherically symmetric, i.e., that $f(U x, U v)=f(x, v)$ for every proper rotation $U$ on $\mathbb{R}^{3}$. It is known then that the solution $f(x, v, t)$ satisfies the same property in $x$ and $v$, and therefore depends only on $r \equiv|x|, u \equiv|v|, \alpha$, and $t$ (cf. [3], [8]) where $\alpha$, the angle between $x$ and $v$, is defined by $x \cdot v=r u \cos \alpha$. The density $\rho$ depends then only on $r$ and $t$ (cf. [3]): $\rho(r, t)=2 \pi \int_{0}^{\infty} \int_{0}^{\pi} f(r, u, \alpha, t) u^{2} \sin \alpha d \alpha d u$, and thus $u$ is also radial. In fact, $u(r, t)=-1 / r \int_{0}^{r} \lambda^{2} \rho(\lambda, t) d \lambda-\int_{r}^{\infty} \lambda \rho(\lambda, t) d \lambda$ by a well-known classical formula. Therefore the electrostatic force $E$ takes the form

$$
E(x, t)=\nabla_{x} u=\frac{x}{r^{3}} \int_{0}^{r} \lambda^{2} \rho(\lambda, t) d \lambda .
$$

It is convenient to introduce the notation

so

$$
M(r, t)=\int_{0}^{r} \lambda^{2} \rho(\lambda, t) d \lambda
$$

$$
E(x, t)=r^{-2} M(r, t) \frac{x}{r}
$$

Note that $\lim _{r \rightarrow \infty} M(r, t)=M / 4 \pi$ and that $|E|=r^{-2} M(r, t)$.

Spherical symmetry also results in a simplification of the characteristic equations:

$$
\left\{\begin{array}{l}
\frac{d R}{d s}=\frac{U \cos A}{\sqrt{1+U^{2}}} \\
\frac{d U}{d s}=\gamma \frac{\cos A}{R^{2}} M(R, s) \\
\frac{d A}{d s}=-\left(\gamma \frac{M(R, s)}{R^{2} U}+\frac{U}{R \sqrt{1+U^{2}}}\right) \sin A
\end{array}\right.
$$


with the initial values $\left.R(s, t, r, u, \alpha)\right|_{s=t}=r,\left.U(s, t, r, u, \alpha)\right|_{s=t}=u$, and $\left.A(s, t, r, u, \alpha)\right|_{s=t}=$ $\alpha$. It follows from (8) that $R U \sin A=r u \sin \alpha$ for all $s$, which is conservation of angular momentum.

\section{Velocity Bounds}

By adapting the methods of Batt [3] and Wollman [13] we may obtain time independent bounds on the velocity in the stellar dynamics case $(\gamma=-1)$ for small radial data, and in the plasma physics case $(\gamma=+1)$ for radial data of unrestricted size. The bound for the plasma physics case uses energy estimates similar to those used by Horst in [8]. It is easily seen, and discussed briefly below, that these velocity bounds imply global existence for (RVP) as they do for (VP) (see Batt [3]). It is shown in Sect. III that no global classical solution can exist in the case $\gamma=-1$ for unrestricted radial data.

We show now why velocity-support bounds on $f(x, v, t)$ imply global classical existence. There are two ways to proceed.

Firstly, in the preprint [7] (to appear), global classical solutions to the full relativistic Vlasov-Maxwell system are found, provided such a velocity bound is known. If such a bound is known a priori, as is the case here, we obtain global solutions for the special case $B \equiv 0$.

Secondly, one may go through Batt's paper [3], and notice that the proof of the global existence theorem remains unchanged up through p. 349 [3]. This is because Poisson's equation $\Delta u=\rho$ is analyzed there, and it is only assumed that $\rho \in L^{1}$, as is the case here. The only change appears when one computes the variational equations for $q_{i k}(s) \equiv \partial / \partial x_{k} X_{i}(s, t, x, v)$ and $p_{i k}(s) \equiv \partial / \partial x_{k} V_{i}(s, t, x, v)$. For (RVP), the variational system is easily seen to be

$$
\dot{q}_{i k}=\frac{p_{i k}-\hat{v}_{i} \sum_{j} \hat{v}_{j} p_{j k}}{\sqrt{1+|v|^{2}}}, \quad \dot{p}_{i k}=\gamma \sum_{j} \frac{\partial E^{i}}{\partial X_{j}} q_{j k} .
$$

Therefore $\sum_{i}\left|\dot{q}_{i k}\right| \leqq 4 \sum_{i}\left|p_{i k}\right|$, and this bound suffices to complete Batt's argument on p. 350 of [3]. A similar variational system arises on p. 353 of [3], and is dealt with in the same manner. Therefore, the result of [3], slightly modified as above for (RVP), shows that a velocity-support bound on $f$ is sufficient for global classical existence. We refer here also to [8], where existence of energy-conserving classical solutions is also obtained for (VP).

Assume $f$ is smooth, nonnegative, spherically symmetric, and vanishes for $(r, u, \alpha) \notin(0, \infty) \times(0, \infty) \times(0, \pi)$. Assume also that $f$ is a classical solution of $(\mathrm{RVP})$ on some time interval $[0, T)$ with initial value $f$. We define for $t \geqq 0$,

$$
P(t)=\sup \{U(s, 0, r, u, \alpha): 0 \leqq s \leqq t, \quad(r, u, \alpha) \in \operatorname{support} \stackrel{\circ}{f}\},
$$

and note that $P$ is nondecreasing. Suppose $u>P(t)$; then we claim that $f(r, u, \alpha, t)=0$. We may set $r_{0}=R(0, t, r, u, \alpha), u_{0}=U(0, t, r, u, \alpha)$, and $\alpha_{0}=A(0, t, r, u, \alpha)$, so that $r=R\left(t, 0, r_{0}, u_{0}, \alpha_{0}\right), \quad u=U_{0}\left(t, 0, r_{0}, u_{0}, \alpha_{0}\right)$, and $\alpha=A\left(t, 0, r_{0}, u_{0}, \alpha_{0}\right)$. Then $u=$ $U\left(t, 0, r_{0}, u_{0}, \alpha_{0}\right)>P(t)$ so $f\left(r_{0}, u_{0}, \alpha_{0}\right)=0$. Hence $f(r, u, \alpha, t)=f\left(r_{0}, u_{0}, \alpha_{0}\right)=0$. This 
observation and the assumptions made above will be used throughout this section.

Lemma I. There exists a constant $C_{1}$ such that for $r \geqq 0$ and $0 \leqq t<T$

$$
|E(x, t)|=\frac{M(r, t)}{r^{2}} \leqq\left\{\begin{array}{l}
\min \left(M r^{-2}, 100 M^{1 / 3}\|\stackrel{\circ}{f}\|_{\infty}^{2 / 3} P^{2}(t)\right) \quad \text { if } \gamma=-1 \\
\min \left(M r^{-2}, C_{1} P^{5 / 3}(t)\right) \text { if } \gamma=+1
\end{array}\right.
$$

Proof: It follows from (7) that $|E(x, t)| \leqq M r^{-2}$ in both cases. Let $R>0$, and note that

$$
\begin{aligned}
|E(x, t)| & =\left|\frac{1}{4 \pi} \int_{\mathbb{R}^{3}} \frac{\rho(y, t)(x-y)}{|x-y|^{3}} d y\right| \leqq \frac{1}{4 \pi} \int_{\mathbb{R}^{3}} \frac{\rho(y, t)}{|x-y|^{2}} d y \leqq \int_{\mathbb{R}^{3}} \frac{\rho(y, t)}{|x-y|^{2}} d y \\
& =\int_{|x-y|<R} \frac{\rho(y, t)}{|x-y|^{2}} d y+\int_{|x-y|>R} \frac{\rho(y, t)}{|x-y|^{2}} d y \\
& \leqq\|\rho(t)\|_{\infty} \int_{|x-y|<R}|x-y|^{-2} d y+R^{-2} \int_{|x-y|>R} \rho(y, t) d y \\
& \leqq 4 \pi R\|\rho(t)\|_{\infty}+M R^{-2} .
\end{aligned}
$$

Taking $R=\left(\|\rho(t)\|_{\infty} / M\right)^{-1 / 3}$, we get $|E(x, t)| \leqq(4 \pi+1) M^{1 / 3}\|\rho(t)\|_{\infty}^{2 / 3}$. But

$$
\rho(r, t)=\int f d v=\int_{|v|<P(t)} f d v \leqq\|f(t)\|_{\infty} \int_{|v|<P(t)} d v=\|\stackrel{\circ}{f}\|_{\infty} \frac{4 \pi}{3} P^{3}(t),
$$

so

$$
|E(x, t)| \leqq(4 \pi+1) M^{1 / 3}\left(\|\stackrel{\circ}{f}\|_{\infty} \frac{4 \pi}{3} P^{3}(t)\right)^{2 / 3} \leqq 100 M^{1 / 3}\|\stackrel{\circ}{f}\|_{\infty}^{2 / 3} P^{2}(t) .
$$

Now repeat this argument using Hölder's inequality and assuming $\gamma=+1$ :

$$
\begin{aligned}
|E(x, t)| & \leqq \int_{|x-y|<R} \frac{\rho(y, t)}{|x-y|^{2}} d y+\int_{|x-y|>R} \frac{\rho(y, t)}{|x-y|^{2}} d y \\
& \leqq\|\rho(t)\|_{\infty} \int_{|x-y|<R}|x-y|^{-2} d y+\|\rho(t)\|_{4 / 3}\left(\int_{|x-y|>R}\left(|x-y|^{-2}\right)^{4} d y\right)^{1 / 4} \\
& =\|\rho(t)\|_{\infty} 4 \pi R+\|\rho(t)\|_{4 / 3}\left(\frac{4 \pi}{5} R^{-5}\right)^{1 / 4}
\end{aligned}
$$

Taking $R=\left(\|\rho(t)\|_{4 / 3} /\|\rho(t)\|_{\infty}\right)^{4 / 9}$, we have

$$
|E(x, t)| \leqq\left(4 \pi+\left[\frac{4 \pi}{5}\right]^{1 / 4}\right)\|\rho(t)\|_{4 / 3}^{4 / 9}\|\rho(t)\|_{\infty}^{5 / 9} .
$$

Now using Proposition I and (10),

$$
|E(x, t)| \leqq\left(4 \pi+\left[\frac{4 \pi}{5}\right]^{1 / 4}\right) C\left(\frac{4 \pi}{3}\|\stackrel{\circ}{f}\|_{\infty} P^{3}(t)\right)^{5 / 9}=C P^{5 / 3}(t),
$$

which completes the proof.

Now we use the method of Batt to treat the case $\gamma=-1$. Fix $T_{0} \in(0, T)$. By 
Lemma I for $t \in\left[0, T_{0}\right]$,

$$
\begin{aligned}
r^{-2} M(r, t) & \leqq \min \left(M r^{-2}, 100 M^{1 / 3}\|\stackrel{\circ}{f}\|_{\infty}^{2 / 3} P^{2}(t)\right) \\
& \leqq \min \left(M r^{-2}, 100 M^{1 / 3}\left\|f^{\circ}\right\|_{\infty}^{2 / 3} P^{2}\left(T_{0}\right)\right) \leqq(A+B r)^{-2}
\end{aligned}
$$

where

$$
B=\frac{1}{2} M^{-1 / 2}
$$

and

$$
A=\frac{1}{2}\left(100 M^{1 / 3}\|\stackrel{\circ}{f}\|_{\infty}^{2 / 3} P^{2}\left(T_{0}\right)\right)^{-1 / 2} .
$$

Let us consider a characteristic through some point $\left(r_{0}, u_{0}, \alpha_{0}\right)$ with $r_{0} u_{0}$ $\sin \alpha_{0}>0$. For notational convenience we denote $R(s)=R\left(s, 0, r_{0}, u_{0}, \alpha_{0}\right)$, $U(s)=U\left(s, 0, r_{0}, u_{0}, \alpha_{0}\right)$, and $A(s)=A\left(s, 0, r_{0}, u_{0}, \alpha_{0}\right)$. Also

$$
L=R(s) U(s) \sin A(s) \text { is a positive constant. }
$$

Define $\xi:[0, \infty) \rightarrow \mathbb{R}$ by

$$
\xi(u)=\sqrt{1+u^{2}}-\int_{L / u}^{\infty}(A+B \eta)^{-2} d \eta \text { for } u>0 \text { and } \xi(0)=1 .
$$

Then $\xi$ is $C^{2}$ on $[0, \infty)$, in fact $\xi^{\prime \prime}(u)=\left(1+u^{2}\right)^{-3 / 2}+2 A L(A u+B L)^{-3}$. So $\xi^{\prime \prime}(u)>0$ for $u \geqq 0$.

Comment. There exists a unique positive $u_{0}$ such that $\xi\left(u_{0}\right)=1$ and $\xi$ is monotonically increasing on $\left(u_{0}, \infty\right) . \xi^{-1}$ will denote the inverse of $\xi:\left[u_{0}, \infty\right) \rightarrow[1, \infty)$.

Lemma II. For all $z \geqq 1$,

$$
\xi^{-1}(z) \leqq\left[\left(z+A^{-1} B^{-1}\right)^{2}-1\right]^{1 / 2} .
$$

Proof. By (15) note that

$$
\begin{aligned}
\xi(u) & =\sqrt{1+u^{2}}-\int_{L / u}^{\infty}(A+B \eta)^{-2} d \eta=\sqrt{1+u^{2}}-B^{-1} u(A u+B L)^{-1} \\
& \geqq \sqrt{1+u^{2}}-A^{-1} B^{-1} \text { for all } u>0 .
\end{aligned}
$$

For any $z \geqq 1$,

so

$$
z=\xi\left(\xi^{-1}(z)\right) \geqq \sqrt{1+\left(\xi^{-1}(z)\right)^{2}}-A^{-1} B^{-1}
$$

and

$$
\left(z+A^{-1} B^{-1}\right)^{2} \geqq 1+\left(\xi^{-1}(z)\right)^{2}
$$

$$
\left[\left(z+A^{-1} B^{-1}\right)^{2}-1\right]^{1 / 2} \geqq \xi^{-1}(z) .
$$

Lemma III. For all $t \in\left[0, T_{0}\right]$,

$$
U(t) \leqq U(0)+\xi^{-1}\left(\sqrt{1+U^{2}(0)}\right), \text { when } \gamma=-1 .
$$

Proof. Let $t \in\left[0, T_{0}\right]$. Suppose $\pi / 2<A(t)$, and define

$$
t_{1}=\inf \{\tau \in[0, t]: \pi / 2<A(s) \text { for all } s \in[\tau, t]\} .
$$


Then $A(s) \geqq \pi / 2$ on $\left[t_{1}, t\right]$, so $\dot{U}(s)=-\left(M(R, s) / R^{2}\right) \cos A \geqq 0$ on $\left[t_{1}, t\right]$, and hence $U(t) \geqq U\left(t_{1}\right)$.

Note that by (8) and (11)

$$
\begin{aligned}
& \frac{d}{d s}\left(\sqrt{1+U^{2}(s)}-\int_{R(s)}^{\infty}(A+B \eta)^{-2} d \eta\right) \\
& =\frac{U \dot{U}}{\sqrt{1+U^{2}}}+\frac{\dot{R}}{(A+B R)^{2}} \\
& =\frac{U \cos A}{\sqrt{1+U^{2}}}\left[(A+B R)^{-2}-\frac{M(R, s)}{R^{2}}\right] \leqq 0 \text { on }\left[t_{1}, t\right] .
\end{aligned}
$$

Note also that $L=R(t) U(t) \sin A(t)<R(t) U(t)$, so $L / U(t)<R(t)$. Combining these we have

$$
\begin{aligned}
\sqrt{1+U^{2}\left(t_{1}\right)}-\int_{R\left(t_{1}\right)}^{\infty}(A+B \eta)^{-2} d \eta & \geqq \sqrt{1+U^{2}(t)}-\int_{R(t)}^{\infty}(A+B \eta)^{-2} d \eta \\
& >\sqrt{1+U^{2}(t)}-\int_{L / U(t)}^{\infty}(A+B \eta)^{-2} d \eta=\xi(U(t))
\end{aligned}
$$

by definition (15).

If $A\left(t_{1}\right)=\pi / 2$, then $R\left(t_{1}\right)=L / U\left(t_{1}\right)$, so (16) becomes $\xi\left(U\left(t_{1}\right)\right) \geqq \xi(U(t))$. Recall that $U(t) \geqq U\left(t_{1}\right)$ and $\xi$ is increasing on $\left(\xi^{-1}(1), \infty\right)$ so $U(t) \leqq \xi^{-1}(1)$.

If $A\left(t_{1}\right)>\pi / 2$, then $t_{1}=0$, and so (16) yields

$$
\xi(U(t)) \leqq \sqrt{1+U^{2}(0)}-\int_{R(0)}^{\infty}(A+B \eta)^{-2} d \eta \leqq \sqrt{1+U^{2}(0)} .
$$

Hence $U(t) \leqq \xi^{-1}\left(\sqrt{1+U^{2}(0)}\right)$. In both cases $\left(A\left(t_{1}\right)=\pi / 2\right.$ and $\left.A\left(t_{1}\right)>\pi / 2\right)$,

$$
U(t) \leqq \xi^{-1}\left(\sqrt{1+U^{2}(0)}\right)
$$

Suppose $A(t) \leqq \pi / 2$ and define

$$
t_{2}=\inf \{\tau \in[0, t]: A(s) \leqq \pi / 2 \text { for all } s \in[\tau, t]\}
$$

$A(s) \leqq \pi / 2 \quad$ on $\quad\left[t_{2}, t\right]$, so $\quad \dot{U}(s)=-M(R, s) \cos A / R^{2} \leqq 0 \quad$ on $\quad\left[t_{2}, t\right]$, and hence $U(t) \leqq U\left(t_{2}\right)$.

If $t_{2}=0, U(t) \leqq U(0)$. Suppose $t_{2} \neq 0$, then there exists a sequence $\left\{t_{2}^{i}\right\}$ such that $0 \leqq t_{2}^{i}<t, t_{2}^{i} \rightarrow t_{2}$ as $i \rightarrow \infty$, and $A\left(t_{2}^{i}\right)>\pi / 2$. By (17) $U\left(t_{2}^{i}\right) \leqq \xi^{-1}\left(\sqrt{1+U^{2}(0)}\right)$, so by continuity $U\left(t_{2}\right) \leqq \xi^{-1}\left(\sqrt{1+U^{2}(0)}\right)$. In both of these cases $\left(t_{2}=0\right.$ or $\left.t_{2} \neq 0\right)$

$$
U(t) \leqq U\left(t_{2}\right) \leqq U(0)+\xi^{-1}\left(\sqrt{1+U^{2}(0)}\right) .
$$

Therefore by $(17)$ and $(18), U(t) \leqq U(0)+\xi^{-1}\left(\sqrt{1+U^{2}(0)}\right)$, which completes the proof.

Theorem I. Let fbe a classical solution of (RVP) on some time interval $[0, T)$ with $\gamma=$ -1 and smooth, nonnegative, spherically symmetric data $f$ which has compact support and vanishes for $(r, u, \alpha) \notin(0, \infty) \times(0, \infty) \times(0, \pi)$. If $40 M^{2 / 3}\|\stackrel{\circ}{f}\|_{\infty}^{1 / 3}<1$, then $P(t)$ (as 
defined in (9)) is uniformly bounded on [0,T), and hence (RVP) possesses a global classical solution.

Proof. By Lemmas II and III,

$$
U(t) \leqq U(0)+\xi^{-1}\left(\sqrt{1+U^{2}(0)}\right) \leqq U(0)+\left[\left(\sqrt{1+U^{2}(0)}+A^{-1} B^{-1}\right)^{2}-1\right]^{1 / 2}
$$

for all $t \in\left[0, T_{0}\right]$. From (9),

$$
\begin{aligned}
P(t) & =\sup \{U(s, 0, r, u, \alpha): 0 \leqq s \leqq t,(r, u, \alpha) \in \operatorname{support} \stackrel{\circ}{f}\} \\
& \leqq P(0)+\left[\left(\sqrt{1+P^{2}(0)}+A^{-1} B^{-1}\right)^{2}-1\right]^{1 / 2} \\
& \leqq P(0)+\sqrt{1+P^{2}(0)}+A^{-1} B^{-1} \text { for } t \in\left[0, T_{0}\right] .
\end{aligned}
$$

Recall the definitions of $A$ and $B\left((12)\right.$ and (13)) to get $P\left(T_{0}\right) \leqq P(0)+\sqrt{1+P^{2}(0)}$ $+40 M^{2 / 3}\|\stackrel{\circ}{f}\|_{\infty}^{1 / 3} P\left(T_{0}\right)$. If $40 M^{2 / 3}\|\stackrel{\circ}{f}\|_{\infty}^{1 / 3}<1$, then $P\left(T_{0}\right)$ cannot exceed a certain constant depending only on $P(0)$ and $M^{2 / 3}\|\stackrel{\circ}{f}\|_{\infty}$. Now since $T_{0}$ was an arbitrary element of $[0, T)$, the theorem follows.

To treat the case $\gamma=+1$ we define

$$
G(r, t)=-\int_{r}^{\infty} \min \left(M \lambda^{-2}, C_{1} P^{5 / 3}(t)\right) d \lambda
$$

for $r \geqq 0$ and $t \geqq 0$. Note that $G$ is continuously differentiable in $r$ and increasing in $r$. Also, letting $R=M^{1 / 2}\left(C_{1} P^{5 / 3}(t)\right)^{-1 / 2}$, we compute

$$
\begin{aligned}
G(0, t) & =-\int_{0}^{R} C_{1} P^{5 / 3}(t) d \lambda-\int_{R}^{\infty} M \lambda^{-2} d \lambda=-C_{1} P^{5 / 3}(t) R-M R^{-1} \\
& =-2 M^{1 / 2}\left(C_{1} P^{5 / 3}(t)\right)^{1 / 2} .
\end{aligned}
$$

We summarize these comments and restate Lemma I with the following:

Comment. $|E(x, t)|=r^{-2} M(r, t) \leqq \partial G / \partial r(r, t)$, and there exists a positive constant $C_{2}$ such that $\left|G\left(r_{1}, t\right)-G\left(r_{2}, t\right)\right| \leqq C_{2} P^{5 / 6}(t)$ for all $r_{1} \geqq 0, r_{2} \geqq 0$, and $t \geqq 0$.

As before we consider a characteristic $R(s)=R\left(s, 0, r_{0}, u_{0}, \alpha_{0}\right), \quad U(s)=$ $U\left(s, 0, r_{0}, u_{0}, \alpha_{0}\right)$, and $A(s)=A\left(s, 0, r_{0}, u_{0}, \alpha_{0}\right)$ through a point $\left(r_{0}, u_{0}, \alpha_{0}\right)$ with $r_{0} u_{0} \sin \alpha_{0} \neq 0$.

Lemma IV. Assume either $\dot{R} \geqq 0$ on $\left[t_{1}, t_{2}\right]$ or $\dot{R} \leqq 0$ on $\left[t_{1}, t_{2}\right]$. Then

$$
\left|\sqrt{1+U^{2}\left(t_{2}\right)}-\sqrt{1+U^{2}\left(t_{1}\right)}\right| \leqq\left|G\left(R\left(t_{2}\right), t_{2}\right)-G\left(R\left(t_{1}\right), t_{2}\right)\right|, \text { for } \gamma=+1 \text {. }
$$

Proof. Note that

$$
\left|G\left(R\left(t_{2}\right), t_{2}\right)-G\left(R\left(t_{1}\right), t_{2}\right)\right|=\left|\int_{t_{1}}^{t_{2}} \frac{\partial G}{\partial r}\left(R(s), t_{2}\right) \dot{R}(s) d s\right|=\int_{t_{1}}^{t_{2}}\left|\frac{\partial G}{\partial r}\left(R(s), t_{2}\right) \dot{R}(s)\right| d s
$$

since $\dot{R}$ is of one sign on $\left[t_{1}, t_{2}\right]$ and $\partial G / \partial r \geqq 0$.

Note also that for $t_{2} \geqq s$,

$$
\frac{\partial G}{\partial r}\left(R(s), t_{2}\right)=\min \left(M R^{-2}(s), C_{1} P^{5 / 3}\left(t_{2}\right)\right) \geqq \min \left(M R^{-2}(s), C_{1} P^{5 / 3}(s)\right)=\frac{\partial G}{\partial r}(R(s), s) .
$$


Now by the characteristic equations, (8), and by the comment

$$
\begin{aligned}
\left|\frac{\partial G}{\partial r}\left(R(s), t_{2}\right) \dot{R}(s)\right| & \geqq\left|\frac{\partial G}{\partial r}(R(s), s) \dot{R}(s)\right| \\
& \geqq\left|\frac{M(R(s), s)}{R^{2}(s)} \frac{U(s) \cos A(s)}{\sqrt{1+U^{2}(s)}}\right|=\left|\frac{U(s) \dot{U}(s)}{\sqrt{1+U^{2}(s)}}\right| \\
& =\left|\frac{d}{d s} \sqrt{1+U^{2}(s)}\right| .
\end{aligned}
$$

Therefore

$$
\begin{aligned}
\left|G\left(R\left(t_{2}\right), t_{2}\right)-G\left(R\left(t_{1}\right), t_{2}\right)\right| & \geqq \int_{t_{1}}^{t_{2}}\left|\frac{d}{d s} \sqrt{1+U^{2}(s)}\right| d s \\
& \geqq\left|\sqrt{1+U^{2}\left(t_{2}\right)}-\sqrt{1+U^{2}\left(t_{1}\right)}\right|,
\end{aligned}
$$

which completes the proof.

Lemma V. Assume $\gamma=+1$. $\dot{R}$ can be zero for at most one value of $s$. If $\dot{R}\left(t_{1}\right)=0$, then $R$ has an absolute minimum at $t_{1}$.

Proof. Recall that $R(s) U(s) \sin A(s)=r_{0} u_{0} \sin \alpha_{0} \neq 0$ by hypothesis, so $R(s) \neq 0$, $U(s) \neq 0$, and $\sin A(s) \neq 0$ for all $s$. From (8),

$$
\dot{R}(s)=\frac{U(s) \cos A(s)}{\sqrt{1+U^{2}(s)}}, \text { so } \quad \dot{R}(s)=0
$$

only if $A(s)=\pi / 2$. But also from (8)

$$
\dot{A}(s)=-\left(\frac{M(R, s)}{R^{2}(s) U(s)}+\frac{U(s)}{R(s) \sqrt{1+U^{2}(s)}}\right) \sin A(s)<0
$$

for all $s$. So $A$ is strictly decreasing for all $s$, and hence can attain the value of $\pi / 2$ at most once. Therefore $\dot{R}$ can be zero at most once.

Suppose $\dot{R}\left(t_{1}\right)=0$, then $A(s)>\pi / 2$ for $s<t_{1}, A\left(t_{1}\right)=\pi / 2$, and $A(s)<\pi / 2$ for $s>t_{1}$. But from (8),

$$
\dot{R}(s)=\frac{U(s) \cos A(s)}{\sqrt{1+U^{2}(s)}}
$$

so $\dot{R}(s)<0$ for $s<t_{1}, \dot{R}\left(t_{1}\right)=0$, and $\dot{R}(s)>0$ for $s>t_{1}$. Therefore $R$ has an absolute minimum at $t_{1}$.

Theorem II. Let fbe a classical solution of (RVP) on some time interval $[0, T)$ with $\gamma=$ +1 and smooth, nonnegative, spherically symmetric data $f$ which has compact support and vanishes for $(r, u, \alpha) \notin(0, \infty) \times(0, \infty) \times(0, \pi)$. Then $P(t)$ (as defined in (9)) is uniformly bounded on $[0, T)$, and hence (RVP) possesses a global classical solution. Proof. Let $t \in[0, T)$. Suppose $0 \leqq t_{1} \leqq t_{2} \leqq t$, and either $\dot{R} \geqq 0$ on $\left[t_{1}, t_{2}\right]$ or $\dot{R} \leqq 0$ on 
$\left[t_{1}, t_{2}\right]$. By Lemma IV,

$$
\sqrt{1+U^{2}\left(t_{2}\right)} \leqq \sqrt{1+U^{2}\left(t_{1}\right)}+\left|G\left(R\left(t_{2}\right), t_{2}\right)-G\left(R\left(t_{1}\right), t_{2}\right)\right|
$$

Now by the comment preceding Lemma IV,

$$
\sqrt{1+U^{2}\left(t_{2}\right)} \leqq \sqrt{1+U^{2}\left(t_{1}\right)}+C_{2} P^{5 / 6}\left(t_{2}\right)
$$

Now if $\dot{R}$ is never zero on $[0, t]$, then by $(20)$,

$$
\sqrt{1+U^{2}(t)} \leqq \sqrt{1+U^{2}(0)}+C_{2} P^{5 / 6}(t)
$$

Now suppose $\dot{R}$ vanishes somewhere on $[0, t]$. By Lemma $V$ there is only one point where $\dot{R}$ vanishes, call it $t_{1} \in[0, t]$. Then $\dot{R}$ cannot change sign on $\left[0, t_{1}\right]$ or on $\left[t_{1}, t\right]$. Hence by applying (20) twice we have

$$
\begin{aligned}
\sqrt{1+U^{2}(t)} \leqq \sqrt{1+U^{2}\left(t_{1}\right)} & +C_{2} P^{5 / 6}(t) \leqq \sqrt{1+U^{2}(0)}+C_{2} P^{5 / 6}\left(t_{1}\right) \\
& +C_{2} P^{5 / 6}(t) \leqq \sqrt{1+U^{2}(0)}+2 C_{2} P^{5 / 6}(t) .
\end{aligned}
$$

Therefore in both cases (whether $\dot{R}$ vanishes or not) we have for $t \in[0, T$ )

$$
\sqrt{1+U^{2}(t)} \leqq \sqrt{1+U^{2}(0)}+2 C_{2} P^{5 / 6}(t) .
$$

Now since $P(t)=\sup \{U(s, 0, r, u, \alpha): 0 \leqq s \leqq t,(r, u, \alpha) \in$ support $\stackrel{\circ}{f}\}$ (see (9)) we have

$$
P(t) \leqq \sqrt{1+P^{2}(t)} \leqq \sqrt{1+P^{2}(0)}+2 C_{2} P^{5 / 6}(t)
$$

It follows that $P$ is uniformly bounded on $[0, T]$, which completes the proof.

Although we will not pursue this here, we point out that if a background charge distribution were present, a modification of Lemma $V$ would probably be required to extend the theorem to this case.

\section{Blow-up of Radial Solutions}

Let $\gamma=-1$ and consider the equation

$$
(\mathrm{RVP})\left\{\begin{array}{l}
f_{t}+\hat{v} \cdot \nabla_{x} f-E \cdot \nabla_{v} f=0 \\
E=\nabla_{x} u, \quad \Delta u=\rho .
\end{array}\right.
$$

From part (C) of Proposition I, Sect. I, the total energy is

$$
\iint_{\mathbb{R}^{6}} \sqrt{1+|v|^{2}} f d v d x-\frac{1}{2} \int_{\mathbb{R}^{3}}|E|^{2} d x=\text { const } \equiv \mathscr{E}_{0} .
$$

From experience one knows that this bodes ill for global existence (unless the second term $\|E\|_{2}^{2}$ can be dominated by the first, as is the case in classical (VP), where the first term (the kinetic energy) is actually $\frac{1}{2} \iint_{\mathbb{R}^{6}}|v|^{2} f d v d x$ ).

To motivate our nonexistence result, we note that

$$
|E|=\left|\nabla_{x} u\right| \leqq c \int \frac{\rho d y}{|x-y|^{2}} \equiv c r^{-2} * \rho .
$$


By the singular integral inequality [5], we have

$$
\|E\|_{2} \leqq c\left\|r^{-2} * \rho\right\|_{2} \leqq c\|\rho\|_{6 / 5},
$$

and further

$$
\|\rho\|_{6 / 5} \leqq\|\rho\|_{1}^{1 / 3}\|\rho\|_{4 / 3}^{2 / 3} \leqq M^{1 / 3}\|\rho\|_{4 / 3}^{2 / 3}
$$

It follows that

$$
\|E\|_{2}^{2} \leqq c M^{2 / 3}\|\rho\|_{4 / 3}^{4 / 3} .
$$

However, inequality (5) shows that

$$
\rho^{4 / 3}(x, t) \leqq c \int_{\mathbb{R}^{3}} \sqrt{1+|v|^{2}} f d v
$$

holds pointwise.

Inequalities (22) and (23) then show that the kinetic and potential energies in $\mathscr{E}_{0}$ are of the same order of magnitude. Thus one expects global solutions only for small data, as we have shown in Sect. II. Indeed, for large data we have the following result:

Theorem III. Let $\stackrel{\circ}{f}$ be smooth, nonnegative, radial and of compact support on $\mathbb{R}^{6}$. Let $f(x, v, t)$ be a classical solution of (RVP) on an interval $0<t<T$ for which $-\infty<$ $\mathscr{E}_{0}<0 .{ }^{1}$ Then $T<\infty$.

Proof. We begin by deriving the "dilation identity" (cf. [6]),

$$
\frac{d}{d t} \iint_{\mathbb{R}^{6}} x \cdot v f d v d x=\mathscr{E}_{0}-\iint_{\mathbb{R}^{6}} \frac{f d v d x}{\sqrt{1+|v|^{2}}} .
$$

Indeed, a direct calculation using (RVP) gives

$$
\begin{aligned}
\frac{d}{d t} \iint_{\mathbb{R}^{6}} x \cdot v f d v d x= & \iint_{\mathbb{R}^{6}} x \cdot v\left[-\hat{v} \cdot \nabla_{x} f+E \cdot \nabla_{v} f\right] d v d x \\
= & \iint_{\mathbb{R}^{6}} x \cdot v\left(-\nabla_{x} \cdot(f \hat{v})\right) d x d v+\iint_{\mathbb{R}^{6}} x \cdot v\left(\nabla_{v} \cdot(f E)\right) d v d x \\
= & \int_{\mathbb{R}^{6}}\left(\nabla_{x} \cdot(-x \cdot v f \hat{v})+f \hat{v} \cdot \nabla_{x}(x \cdot v)\right) d x d v \\
& +\int_{\mathbb{R}^{6}}\left(\nabla_{v} \cdot(x \cdot v f E)-f E \cdot \nabla_{v}(x \cdot v)\right) d v d x \\
= & \iint_{\mathbb{R}^{6}} f(\hat{v} \cdot v-E \cdot x) d v d x=\iint_{\mathbb{R}^{6}} \frac{|v|^{2} f}{\sqrt{1+|v|^{2}}} d v d x-\int_{\mathbb{R}^{3}} \rho x \cdot E d x .
\end{aligned}
$$

Now since $\Delta u=\rho$ and $E=\nabla_{x} u$, we have

$$
\int_{\mathbb{R}^{3}} \rho x \cdot E d x=\int_{\mathbb{R}^{3}}\left(\sum_{k} u_{x_{k} x_{k}}\right)\left(\sum_{j} x_{j} u_{x_{j}}\right) d x
$$

1 The condition $\mathscr{E}_{0}<0$ is easily achieved by replacing $\stackrel{\circ}{f}$ by $\lambda \dot{f}$, where $\lambda>0$ is chosen sufficiently large 


$$
\begin{aligned}
& =\int_{\mathbb{R}^{3}} \sum_{k}\left(\left[u_{x_{k}} \sum_{j} x_{j} u_{x_{j}}\right]_{x_{k}}-u_{x_{k}} \sum_{j} x_{j} u_{x_{j} x_{k}}-u_{x_{k}}^{2}\right) d x \\
& =-\int_{\mathbb{R}^{3}} \sum_{k} \sum_{j} x_{j} \frac{\partial}{\partial x_{j}}\left(\frac{1}{2} u_{x_{k}}^{2}\right) d x-\int_{\mathbb{R}^{3}}|\nabla u|^{2} d x \\
& =-\sum_{k} \int_{\mathbb{R}^{3}} \sum_{j}\left[\frac{\partial}{\partial x_{j}}\left(x_{j} \frac{1}{2} u_{x_{k}}^{2}\right)-\frac{1}{2} u_{x_{k}}^{2}\right] d x-\int_{\mathbb{R}^{3}}|\nabla u|^{2} d x \\
& =\frac{1}{2} \sum_{k} \int_{\mathbb{R}^{3}}|\nabla u|^{2} d x-\int_{\mathbb{R}^{3}}|\nabla u|^{2} d x=\frac{1}{2} \int_{\mathbb{R}^{3}}|\nabla u|^{2} d x .
\end{aligned}
$$

Thus we find

$$
\begin{aligned}
\frac{d}{d t}\left(\iint_{\mathbb{R}^{6}} x \cdot v f d v d x\right) & =\iint_{\mathbb{R}^{6}} \frac{|v|^{2} f}{\sqrt{1+|v|^{2}}} d v d x-\frac{1}{2} \int_{\mathbb{R}^{3}}|E|^{2} d x \\
& =\iint_{\mathbb{R}^{6}}\left(\sqrt{1+|v|^{2}}-\frac{1}{\sqrt{1+|v|^{2}}}\right) f d v d x-\frac{1}{2} \int_{\mathbb{R}^{3}}|E|^{2} d x \\
& =\mathscr{E}_{0}-\iint_{\mathbb{R}^{6}} \frac{1}{\sqrt{1+|v|^{2}}} f d v d x,
\end{aligned}
$$

as claimed.

Given the dilation identity, the proof proceeds as follows: first integration of the dilation identity in $t$ produces

$$
\iint_{\mathbb{R}^{6}} x \cdot v f d v d x \leqq \iint_{\mathbb{R}^{6}} x \cdot v \stackrel{\circ}{f} d v d x+\mathscr{E}_{0} t .
$$

Next, using (RVP) we compute

$$
\begin{aligned}
\frac{d}{d t} \iint_{\mathbb{R}^{6}} r^{2} \sqrt{1+|v|^{2}} f d v d x & =\iint_{\mathbb{R}^{6}} r^{2} \sqrt{1+|v|^{2}}\left[-\hat{v} \cdot \nabla_{x} f+E \cdot \nabla_{v} f\right] d v d x \\
& =-\iint_{\mathbb{R}^{6}} r^{2} v \cdot \nabla_{x} f d v d x+\iint_{\mathbb{R}^{6}} r^{2} \sqrt{1+|v|^{2}} \nabla_{v} \cdot(E f) d v d x \\
& =2 \iint_{\mathbb{R}^{6}} x \cdot v f d v d x-\iint_{\mathbb{R}^{6}} r^{2} f E \cdot \frac{v}{\sqrt{1+|v|^{2}}} d v d x \\
& =2 \iint_{\mathbb{R}^{6}} x \cdot v f d v d x-\int_{\mathbb{R}^{3}} r^{2} E \cdot j d x,
\end{aligned}
$$

where $j=\int_{\mathbb{R}^{3}} \hat{v} f d v$.

We now use the radial nature of the solution: by Lemma $\mathrm{I}|E(x, t)| \leqq M r^{-2}$. Moreover $|j| \leqq \rho$, by definition. Thus for the last term we have the estimate

$$
\left|\int_{\mathbb{R}^{3}} r^{2} E \cdot j d x\right| \leqq M \int_{\mathbb{R}^{3}}|j| d x \leqq M \int_{\mathbb{R}^{3}} \rho d x \leqq M^{2},
$$


so that by (25) and (26)

$$
\begin{aligned}
\frac{d}{d t} \iint_{\mathbb{R}^{6}} r^{2} \sqrt{1+|v|^{2}} f d v d x & \leqq 2 \iint_{\mathbb{R}^{6}} x \cdot v f d v d x+M^{2} \\
& \leqq 2\left(\iint_{\mathbb{R}^{6}} x \cdot v \stackrel{\circ}{f} d v d x+\mathscr{E}_{0} t\right)+M^{2} \\
& =\text { Constant }+2 \mathscr{E}_{0} t .
\end{aligned}
$$

Integrating in time we get

$$
\begin{aligned}
\iint_{\mathbb{R}^{6}} r^{2} \sqrt{1+|v|^{2}} f d v d x \leqq & \iint_{\mathbb{R}^{6}} r^{2} \sqrt{1+|v|^{2}} \stackrel{\circ}{f} d v d x \\
& +\left(2 \iint_{\mathbb{R}^{6}} x \cdot v \stackrel{\circ}{f} d v d x+M^{2}\right) t+\mathscr{E}_{0} t^{2} \\
= & C+C t+\mathscr{E}_{0} t^{2} .
\end{aligned}
$$

Now since $\mathscr{E}_{0}<0$ by hypothesis, the right-hand side here becomes negative for large $t$. The left-hand side is nonnegative so the existence time $T$ must be finite, completing the proof.

\section{References}

1. Arsen'ev, A.: Global existence of a weak solution of Vlasov's system of equations. U.S.S.R. Comput. Math. Math. Phys. 15, 131-143 (1975)

2. Bardos, C., Degond, P.: Global existence for the Vlasov-Poisson equation in three space variables with small initial data. Preprint

3. Batt, J.: Global symmetric solutions of the initial-value problem of stellar dynamics. J. Differ. Equations 25, 342-364 (1977)

4. Batt, J.: The nonlinear Vlasov-Poisson system of partial differential equations in stellar dynamics, Publ. C.N.E.R. Math. Pures Appl. Année 83, Vol. 5, Fasc. 2, 1-30 (Lille, 1983)

5. Bers, L., John, F., Schechter, M.: Partial differential equations. New York: Interscience 1966

6. Glassey, R., Strauss, W.: Remarks on collisionless plasmas. Contemp. Math. 28, 269-279 (1984)

7. Glassey, R., Strauss, W.: Singularity formation in a collisionless plasma could occur only at high velocities. To appear in Arch. Rat. Mech. Anal.

8. Horst, E.: On the classical solutions of the initial value problem for the unmodified non-linear Vlasov equation. Parts I and II. Math. Method Appl. Sci. 3, 229-248 (1981); 4, 19-32 (1982)

9. Horst, E., Hunze, R.: Weak solutions of the initial value problem for the unmodified nonlinear Vlasov equation. Math. Methods Appl. Sci. 6, 262-279 (1984)

10. Ukai, S., Okabe, T.: On classical solutions in the large in time of two-dimensional Vlasov's equation. Osaka J. Math. 15, 245-261 (1978)

11. Van Kampen, N. G. Felderhof, B. V.: Theoretical methods in plasma physics p. 170. Amsterdam: North-Holland 1967

12. Weibel, E.: L'equation de Vlasov dans la theorie speciale de la relativite. Plasma Phys. 9, 665-670 (1967)

13. Wollman, S.: The spherically symmetric Vlasov-Poisson system. J. Differ. Equations 35, 30-35 (1980)

14. Wollman, S.: An existence and uniqueness theorem for the Vlasov-Maxwell system. Commun. Pure Appl. Math. 37, 457-462 (1984)

15. Wollman, S.: Global-in-time solutions of the two-dimensional Vlasov-Poisson system. Commun. Pure Appl. Math. 33, 173-197 (1980)

Communicated by L. Nirenberg

Received December 7, 1984; in revised form April 19, 1985 
